Original article

\title{
High sensitivity to multisensory conflicts in agoraphobia exhibited by virtual reality
}

\author{
Isabelle Viaud-Delmon ${ }^{\mathrm{a}, *}$, Olivier Warusfel ${ }^{\mathrm{b}}$, Angeline Seguelas ${ }^{\mathrm{a}}$, \\ Emmanuel Rio ${ }^{\mathrm{b}}$, Roland Jouvent ${ }^{\mathrm{a}}$ \\ ${ }^{a}$ CNRS - UPMC UMR 7593, Pavillon Clérambault, Hôpital de la Salpêtrière, 47, boulevard de l'Hôpital, 75013 Paris, France \\ ${ }^{\mathrm{b}}$ IRCAM - CNRS UMR 9912, Paris, France
}

Received 7 July 2004; received in revised form 13 October 2004; accepted 21 October 2004

Available online 03 February 2005

\begin{abstract}
The primary aim of this study was to evaluate the effect of auditory feedback in a VR system planned for clinical use and to address the different factors that should be taken into account in building a bimodal virtual environment (VE). We conducted an experiment in which we assessed spatial performances in agoraphobic patients and normal subjects comparing two kinds of VEs, visual alone (Vis) and auditoryvisual (AVis), during separate sessions. Subjects were equipped with a head-mounted display coupled with an electromagnetic sensor system and immersed in a virtual town. Their task was to locate different landmarks and become familiar with the town. In the AVis condition subjects were equipped with the head-mounted display and headphones, which delivered a soundscape updated in real-time according to their movement in the virtual town. While general performances remained comparable across the conditions, the reported feeling of immersion was more compelling in the AVis environment. However, patients exhibited more cybersickness symptoms in this condition. The result of this study points to the multisensory integration deficit of agoraphobic patients and underline the need for further research on multimodal VR systems for clinical use.
\end{abstract}

(C) 2005 Elsevier Masson SAS. All rights reserved.

Keywords: Virtual reality; Multisensory integration; Agoraphobia; Anxiety disorders; Cybertherapy

\section{Introduction}

Virtual reality (VR) integrates real-time computer graphics, body tracking devices and visual displays to immerse a user in a computer-generated virtual environment (VE). Other sensorial interfaces can also be used such as force or tactile feedback systems. All these interfaces enable the user to become an active participant within a virtual world and to give the user a sense of presence in the VE (sense of being in the place depicted by VR rather than in the physical place where the user is located-see [18] for a phenomenological description of presence). The setting in which the user performs an action can be controlled by the experimenter, recorded and measured. The unique features and flexibility of VR give it extraordinary potential for use in human sciences research [30].

\footnotetext{
* Corresponding author. Tel.: +331442307 50; fax: +331537907 70 .

E-mail address: ivd@ext.jussieu.fr (I. Viaud-Delmon).
}

Regarding mental health research, the application of VR technologies are potentially numerous. For instance, VR has been employed as an alternative for in vivo exposure for the treatment of different phobias for the past decade [3,5,21,25]. In order to treat a phobia, the virtual exposure needs to evoke the same reactions and emotions as a real experience. A study on acrophobia reported that presence was the best predictor of fear of heights in a VE [23]. Another study involving patients with different specific phobia demonstrated a positive correlation between emotions and presence [24].

The number of sensory modalities through which the user is coupled to the VE is a main factor contributing to the feeling of presence [27]. The specific feature of VR compared to traditional displays is indeed that the environments it provides are places where as many as possible senses are meant to be active. In spite of that, VR technologies rarely integrate the auditory modality, which is the only sense through which we are communicating with the whole space around us. 
Psychiatric patients commonly report a hypersensitivity to auditory stimuli, while pure tone audiograms show generally normal hearing. Sound tolerance is influenced by stress and tiredness, and specific sounds can cause physical pain and nerve grating [20]. There is a correlation between loudness tolerance and anxiety [29] and strong emotional reactions can easily be elicited through audition [4]. It is therefore all the more interesting to integrate the auditory modality in a realistic way when working with anxious patients in VR and to understand in which way it can be used for therapeutic purposes.

Several studies have led to the observation that patients with agoraphobia, panic or space and motion discomfort (SMD) may have a problem with multisensory integration: subjects with symptoms of panic and agoraphobia are destabilized under conflicting sensory conditions while maintaining upright posture $[12,13,35]$. Previous studies creating conflicts between vestibular and visual information with a VR set-up attempted to demonstrate that abnormal central processes of multisensory integration in anxiety disorders was not restricted to balance control $[32,33]$. If the auditory modality is integrated to a VR set-up, multisensory processes can be addressed in yet another way. Without any additional sensory conflict than the one which is inherent to a VR set-up (due to the delay in feedback between action and consequences of actions in the VE), how do agoraphobic patients cope with interactive auditory modality? Would the introduction of the auditory modality generate sensory enhancement or sensory overload?

The study we present here involves technologies, models and applications linked to the introduction of 3-D sound in virtual or augmented reality environments. Auditory augmentation of visual environments is known to improve presence and immersion [10]. To create such an environment and the corresponding content, several concepts and technologies need to be researched, developed, and/or integrated. The introduction of 3-D sound also addresses the need for a better understanding of multisensory integration mechanisms. This includes complementarities or conflict between the auditory and visual senses and also with idiothetic cues (cues generated through self-motion, including vestibular and proprioceptive information).

The most natural audio technique for VR applications is binaural rendering on headphones that relies on the use of HRTFs (HRTF refers to head related transfer function, which is a set of filters measured on an individual or artificial head and used to reproduce all the directional cues involved in auditory localization [2]). However, incorporating real-time updated 3-D sound to VR technologies addresses several practical issues. If there is a consensus on the fact that presence is improved by 3-D sound [8], little is known about how an auditory VE should be designed so that it does not interfere with the visual VE [31]. We thus conducted a study to provide information about the importance of auditory feedback in a VR system planned for clinical use, as well as about the different factors which should be taken into account to build a multimodal VE (sense of realism, presence and coherence between the visual and auditory VE).

If agoraphobic patients are effectively more sensitive to sensory conflicts than normal subjects [32], then multisensory feedback in VR could represent a challenge for them. However, since presence during a bimodal stimulation should be higher, this might provide an interesting way to convey supplementary spatial information and engage patients in a task. We conducted a study in which we compared navigation performances in a virtual town in two immersive conditions: visual alone (Vis) and auditory-visual (AVis). We intended to test the emotional and behavioral reaction of patients sensitive to space and of normal subjects to these two kinds of virtual stimulation in order to develop new procedures and find an integrated approach to work with vision and audition in VR.

\section{Material and methods}

\subsection{Design}

All subjects included in the comparative study received two sessions of virtual navigation. The order of sessions was counterbalanced; so that the same number of subjects began the trial with AVis and Vis conditions. Sessions were performed at least 1 week apart. After each session, subjects had to complete several questionnaires and two memory tests related to their experience. In the first one, they were presented a survey view of the virtual town and had to locate the different landmarks they were asked to find during navigation. In the second one, they were submitted to a two-choice forced recognition task, during which they were presented 10 pairs of snapshots and were required to chose between a view taken in the virtual town they had navigated in and a view taken in a novel town.

The measures taken during the navigation were the number of landmarks found (score on 11) and the time spent in the virtual town. The measures taken after navigation were the number of correctly localized landmarks on the survey view of the virtual town (score on 12) and the number of correct answers to the two-choice forced recognition task (score on 10). Participants were debriefed after each session but were not informed about the content of the following session. At the end of the second session and after the debriefing they were informed about the differences between the two conditions if they had not noticed it.

\subsection{Procedure}

Subjects were equipped with a head-mounted display coupled with an electromagnetic sensor system and immersed in the virtual town in which they could move forward by pressing a mouse button. Subjects had to turn on their own vertical axis in order to change the direction of heading in the virtual town. Their task was to locate different landmarks (movie 


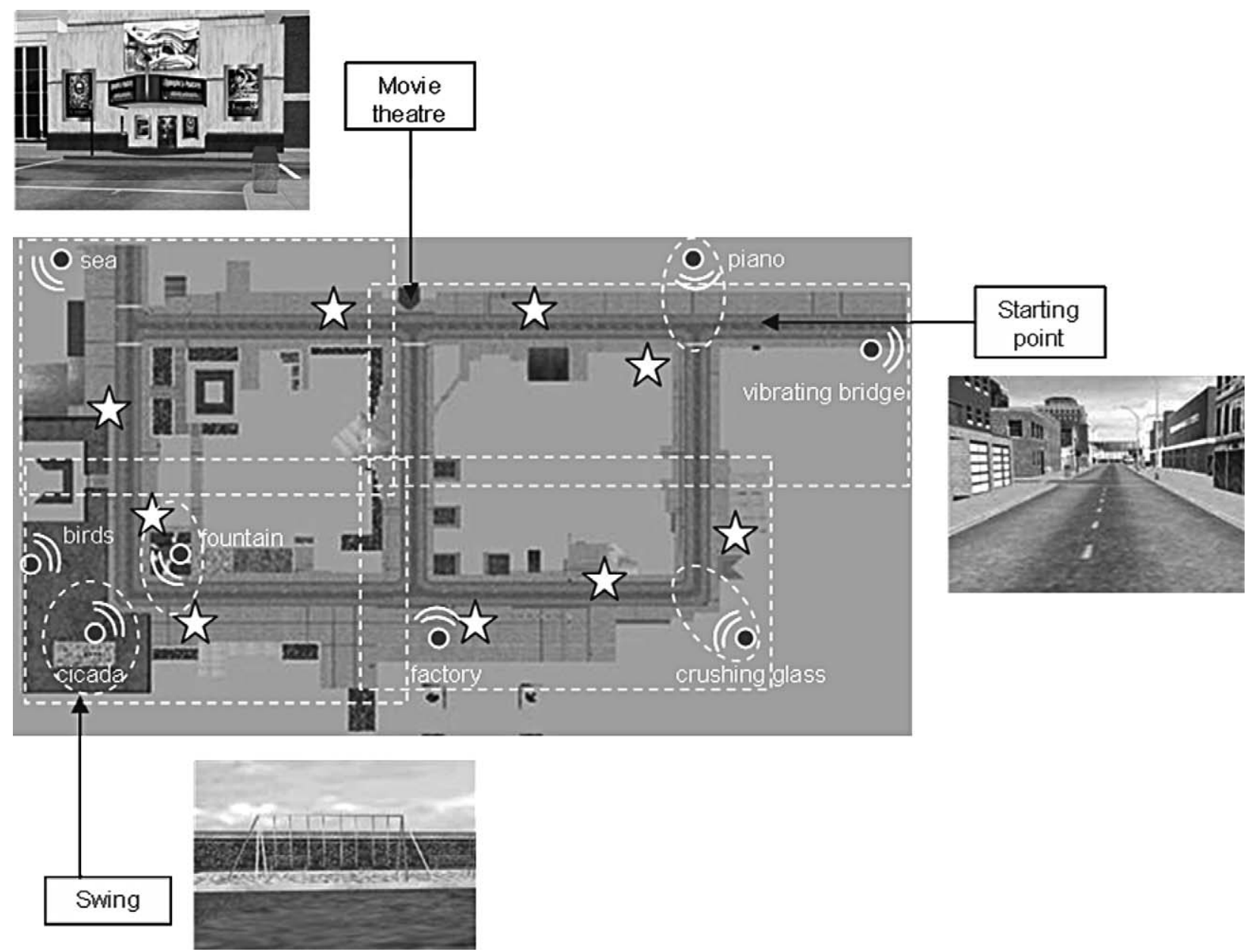

Fig. 1. Survey view of the visual environment in Vis and AVis conditions. The subject's task is to find the movie theater, then the swing, then to count the total number of bus stops (depicted here by stars) in the virtual town. The subject stops navigating when he/she thinks that he/she is familiar with the town and that he/she has localized all the targets. In Avis condition, the sounds (depicted here by small dark circles outlined in white) are played according to the computed position and distance of the subject with respect to the source when he/she enters an activation area. Large activation areas (white dashed lines rectangles): ambisonic sounds scenes (four channels audio format) recorded in an urban environment. Small activation areas (white dashed lines ellipses): binaurally rendered monophonic sources.

theater, swing, bus stops, see Fig. 1) and become familiar with the town. In the AVis condition subjects were equipped with the head-mounted display and headphones, which delivered a soundscape updated in real-time according to their movement in the virtual town. The sounds were produced through tracked binaural rendering (non individual HRTF) and were dependent upon the subject's movements.

\subsection{Subjects}

Patients were individuals diagnosed as affected by agoraphobia with or without panic disorder according to the DSMIV. Seventeen patients were referred to us for the study at the local day hospital ( 12 females, five males). Two of them were excluded from the following analysis since their fear of empty spaces was so severe that they could not accomplish the task. Five patients stopped the protocol after the first session (three females, two males). The remaining 10 patients completed the project (seven females, three males). Nine control subjects (seven females, two males) were included in the study.
The mean ages of the anxious and control samples were 35.3 (S.D. 10.2) and 32.7 years (S.D. 10.6), respectively. The control participants were not afflicted with any mental disorders. A semi-structured interview based on the Mini International Neuropsychiatric Interview [16] was administered to all the participants to ensure that they met the selection criteria. Subjects were also required to complete the trait portion of the STAI [28], the Motion Sickness Questionnaire (susceptibility to motion sickness in different ways of traveling) [22] and the Derealization Experience Scale [1,6]. Informed consent was obtained from all the participants. The study was performed in accordance with the Declaration of Helsinki/Hong Kong.

\subsection{Questionnaires and interview measures}

The state portion of the STAI [28] was used to measure the anxiety levels upon arrival at the laboratory and after completion of the experiment. A 22-item cybersickness scale was used to assess the level of discomfort after exposure to 
VR. It comprised a list of symptoms and sensations associated with autonomic arousal (nausea, sweating, heart pounding, etc.), vestibular symptoms (dizziness, fainting, etc.), respiratory symptoms (feeling short of breath, etc.) and could also be used to estimate signs of somatisation (tendency to complain of a large number of diverse symptoms). Items were rated on a scale from 0 to 4 (absent, weak, moderate, strong). The presence questionnaire from the I-group [26] was presented after completion of the experiment.

\subsection{Visual and auditory stimuli}

The 3-D visual environment was based on a 3-D model developed by Sense8 Corp (San Rafael, CA). It was composed of noticeable landmarks, several streets and alleys and was rendered using Virtools Dev 2.5 (Virtools SA, Paris, France). This software has limited features in terms of auditory design, which consists mainly of stereo rendered sources. In order to allow the maximum flexibility with regards to sound design, we used the Spat sound rendering engine $[14,15]$ and the ListenSpace auditory scene authoring tool [7]; both developed at Ircam. The sounds were produced through tracked binaural rendering (non individual HRTFs) and were dependent upon the subject's movements.

Two types of auditory elements were used; binaurally rendered monophonic sources and ambisonics sound scenes (Fig. 1). Binaurally rendered monophonic sources were put at precise locations in the scene and linked to small activation areas where the sources could be heard. Ambisonics sound scenes, linked to large activation areas, were added to the soundscape. Ambisonics is a four channel audio format that embodies spatial information of a sound scene according to the three directions of space (left/right, front/back and up/down), thus allowing a full immersion of the listener inside an auditory environment. The large activation areas covered the whole town, so that the subject was either at the center of at least one sound scene, or in a cross-fade region between two sound scenes. The cross-fade mechanism was tuned to ensure smooth transitions between the four sound scenes.

\subsection{VR set-up specifications}

We used a V8 head-mounted display (Virtual Research Systems, Santa Clara, CA). The LCD displays had a monocular field of view of $48^{\circ}$ by $36^{\circ}$, with an array of $640 \times 480$ (true VGA) color triads (pixels), refreshed at 60 frames per s. The subject's head orientation was measured by an electromagnetic sensor system (Fastrak Polhemus) which has an update rate of $120 \mathrm{~Hz}$. The image generator (Pentium IV $2.4 \mathrm{GHz}$, $512 \mathrm{mb}$ of RAM and an NVIDIA Quadro4750 XGL graphics card) took the head angular position information from the tracker and sent the corresponding image to the display and to ListenSpace (Pentium IV $2.4 \mathrm{GHz}$ ), which calculated the position of the sound sources with respect to the head angular position information and sent them to the Spat (Mac $1 \mathrm{GHz}$ ), which generated the sound. The Mac was equipped with a Hammerfall DSP system. Sennheiser HD570 circumaural open headphones were used in the AVis condition.

\section{Results}

Of the 17 recruited patients, as mentioned before two females had to be excluded because of strong emotional reactions. Interestingly, the protocol had a beneficial effect for the two of them, who eventually managed to perform part of the navigation task at the end of the second session (with two landmarks found). Five patients completed only one session. Two did an AVis condition (two females), three did a Vis condition (one female, two males). Only the 10 patients who completed two sessions were included in the comparative analysis (see Table 1).

\subsection{Questionnaires measures and VR}

\subsubsection{Presence}

A two-way ANOVA with condition (Vis vs. AVis) as a within-subjects factor and with group (patients vs. control) as between-subjects factor on presence scores indicated a main effect of condition $(F(1,17)=7.3, P=0.01)$.

Presence scores were higher in the AVis condition in both groups of subjects (Table 1). The analysis of variance with condition as a within-subjects factor and with presentation order of conditions as a between-subjects factor indicated only a main effect of condition $(F(1,17)=9.29, P<0.01)$. However, the interaction between presentation order and condition was marginally significant $(F(1,17)=4.1, P=0.06)$.

Table 1

Means and (S.D.) of the scores to the different measures related to VR according to the group and the condition

\begin{tabular}{|c|c|c|c|c|}
\hline \multirow[b]{2}{*}{ Measure } & \multicolumn{2}{|c|}{ Vis condition } & \multicolumn{2}{|c|}{ AVis condition } \\
\hline & $\begin{array}{l}\text { Control group } \\
(n=9)\end{array}$ & $\begin{array}{l}\text { Patients group } \\
(n=10)\end{array}$ & $\begin{array}{l}\text { Control group } \\
(n=9)\end{array}$ & $\begin{array}{l}\text { Patients group } \\
(n=10)\end{array}$ \\
\hline Time in VR (in s) & $474(206)$ & 509 (194) & 469 (170) & $428(211)$ \\
\hline Landmarks found during navigation $(\mathrm{Max}=11)$ & $9.7(2.9)$ & $8.6(2.9)$ & $10.4(1.1)$ & $8.9(3.8)$ \\
\hline Two-choice forced recognition $(\mathrm{Max}=10)$ & $6.9(2.0)$ & $7.7(1.4)^{\mathrm{a}}$ & $7.3(1.1)$ & $6.6(1.6)^{\mathrm{a}}$ \\
\hline Correctly localized landmarks $(\operatorname{Max}=12)$ & $7.3(2.6)^{\mathrm{b}}$ & $5.0(3.3)^{\mathrm{b}}$ & $6.9(2.8)$ & $5.1(3.3)$ \\
\hline Cybersickness & $5.2(5.3)$ & $6(7.8)$ & $4.6(6.2)$ & $18.8(14.6)$ \\
\hline Presence & 32.4 (13.1) & $38(17.2)$ & $40(14.1)$ & $42.6(19.2)$ \\
\hline
\end{tabular}

${ }^{a}$ Wilcoxon rank test, $t=3, P<0.05$.

${ }^{\mathrm{b}}$ Mann-Whitney $U$-test, $z=-2, P<0.05$. 
Indeed, presence scores increased during the second session only in Vis first presentation order. In AVis first presentation order, presence scores decreased during the second session (for Vis first, Vis $=39.6$, S.D. $=15.2$, AVis $=41.7$, S.D. $=18.7$; for AVis first, $\mathrm{AVis}=41$, S.D. $=15$, Vis $=30.7$, S.D. $=14.7$ ).

This observation is in agreement with the finding that auditory modality improves the sense of presence, since removing it has the opposite effect.

Interestingly, the scores from the DES were correlated with the scores from presence scale, both in Vis condition $(r=0.63$, $P<0.01, n=22)$ and in AVis condition $(r=0.48, P<0.05$, $n=21$ ). Presence scores did not correlate with neither of the state anxiety scores or the trait scores.

\subsubsection{Cybersickness}

An ANOVA with condition as a within-subjects factor and with group as a between-subjects factor on cybersickness scores showed an interaction between the factors group and condition $(F(1,17)=10.6, P<0.01)$. Cybersickness scores significantly increased in AVis condition in patients group (Fig. 2). It is unlikely that these scores represent a general tendency towards somatisation since there is no difference between the two groups of subjects in the Vis condition. The analysis of variance with condition as a within-subjects factor and with presentation order of conditions as a betweensubjects factor indicated only a main effect of condition $(F(1,17)=6.1, P<0.05)$.

Cybersickness scores and scores from the state anxiety scale completed after the Vis session (but not before) were correlated ( $r=0.57, P<0.01, n=22)$.

In AVis condition, state anxiety scores both before and after the session correlated with cybersickness scores $(r=0.62$, $P<0.01 ; r=0.83, P<0.0001, n=21)$.

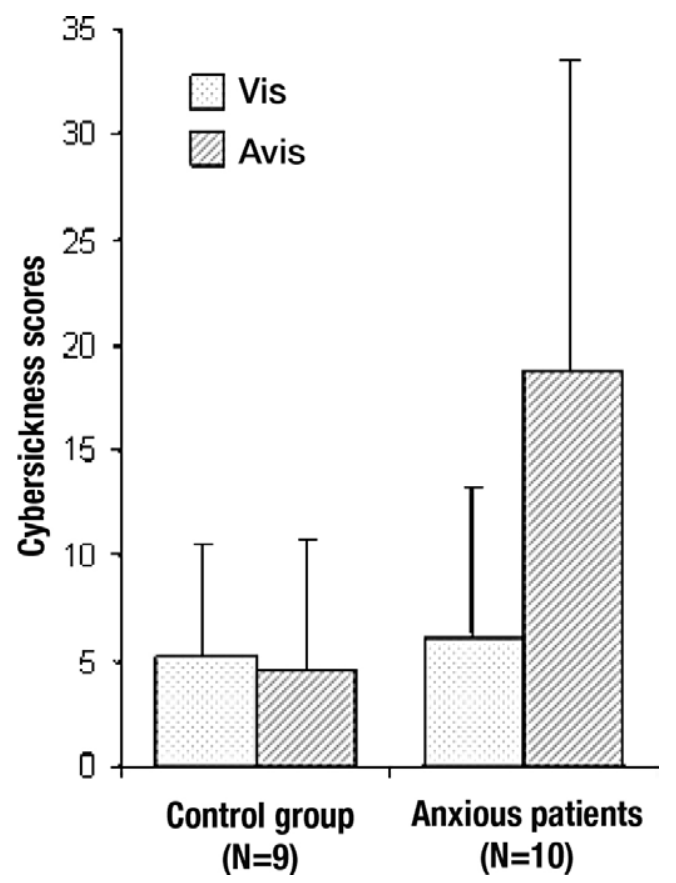

Fig. 2. Cybersickness scores according to the condition and the group. Error bars represent 1 S.D.
Table 2

Means and (S.D.) of state anxiety levels at the beginning and after completing a session

\begin{tabular}{|c|c|c|c|c|}
\hline \multirow[b]{2}{*}{ Time } & \multicolumn{2}{|c|}{ Control group $(n=9)$} & \multicolumn{2}{|c|}{ Patient group $(n=10)$} \\
\hline & $\begin{array}{l}\text { Vis } \\
\text { condition }\end{array}$ & $\begin{array}{l}\text { AVis } \\
\text { condition }\end{array}$ & $\begin{array}{l}\text { Vis } \\
\text { condition }\end{array}$ & $\begin{array}{l}\text { AVis } \\
\text { condition }\end{array}$ \\
\hline Before the session & $23.7(2.7)$ & $23.4(2.7)$ & $29.6(7.6)$ & $33(8.6)$ \\
\hline At the end of session & $22.7(4.2)$ & $24.4(5.1)$ & $32.4(10.2)$ & $37(10.8)$ \\
\hline
\end{tabular}

Cybersickness scores and trait anxiety scores were correlated both in Vis and AVis conditions $(r=0.48, P<0.05, n$ $=22 ; r=0.65, P<0.01, n=21$; respectively). No relationship was found between the general sensitivity to motion sickness [22] and cybersickness scores.

\subsubsection{State anxiety levels}

The two groups differed in all measures of state anxiety (Table 2). A three-way analysis of variance with two repeated measures on condition and the two state anxiety scores (before and after the VR session) was performed between the two groups $(2 \times 2 \times 2)$. The analysis indicated a main effect of group $(F(1,17)=12.9, P<0.01)$ but there was no interaction between the different factors, suggesting that state anxiety levels before and after the VR session were not statistically different for any of the groups.

\subsection{Navigation in Vis and Avis condition}

No difference between the groups nor between the conditions was found in the two measures taken during navigation (time spent in VR and number of landmarks found). The analysis of variance with condition as a within-subjects factor and with presentation order of conditions as a betweensubjects factor indicated an interaction between presentation order of conditions and condition on time spent in VR $(F(1,17)=11.6, P<0.01)$. Indeed, time spent in VR always decreased during the second session, but time spent in VR during the first session was much shorter in the case of AVis first (for Vis first, Vis $=573$, S.D. $=149 \mathrm{~s}$, AVis $=438 \mathrm{~s}$, S.D. $=162$; for $A$ Vis first, $\mathrm{AVis}=462 \mathrm{~s}$, S.D. $=220$, Vis $=403 \mathrm{~s}$, S.D. $=209)$. Since time spent in VR is a measure of time to find the landmarks and to feel familiarity with the town, this indicates that $\mathrm{AVis}$ condition does provide more efficiency in spatial exploration.

\subsection{Correctly localized landmarks (after navigation)}

No difference between the groups or between the conditions was found. The analysis of variance with condition as a within-subjects factor and with presentation order of conditions as a between-subjects factor indicated an interaction between presentation order and condition $(F(1,17)=12.9$ $P<0.01)$. Performance always increased for the second session, but the increase was higher when the first session was in AVis condition (for Vis first, Vis $=5.1$, S.D. $=3.3$, AVis $=6.5$, S.D. $=3.1$; for AVis first, $\mathrm{AVis}=5.3$, S.D. $=3.3$, Vis = 7.2, S.D. $=2.7)$. 


\subsection{Two-choice forced recognition (after navigation)}

No difference between the groups or between the conditions was found with either analysis of variance. However, the interaction between group and condition was marginally significant $(F(1,17)=3.04, P=0.09)$. While normal subjects' performance tended to increase in AVis condition (see Table 1), patients' performance decreased in comparison with performance in Vis condition (Wilcoxon rank test, $t=3, P$ $<0.05)$. No presentation order effect could explain this observation.

\section{Discussion}

In the present experiment, we compared navigation in a visual VE with navigation in an auditory-visual VE in two samples of subjects. As expected, presence scores were significantly higher in AVis condition. Presentation order of conditions was not at stake in this result, since the order of sessions was counterbalanced across subjects. However, analysis of the effect of presentation order pointed to a beneficial effect of auditory modality: time spent in VR to find the landmarks was shorter in AVis first order, the increase of number of correctly localized landmarks at the end of the second session was higher in AVis first order.

In general, the goal to achieve in immersion in a VE is to give to the user a compelling illusion that he actually moves in the VE and no longer in the physical world. If the virtual frame of reference is completely adopted, the user will describe a high degree of presence. Paradoxically, this feeling would refer to a derealization experience, since it would involve an alteration in the perception of the external world which would not seem more real anymore than the virtual world. The correlation found between presence scores and dissociation scores points indeed to a link between the two states, especially since this relationship has already been reported in at least one other study [34].

It remains to be proved whether this relationship could constitute a risk or a benefice for patients; the latter being that a strong sense of presence would facilitate emotional reactions in the VE hence would be beneficial for therapy; the former being that it might be difficult for some patients to distinguish afterwards between the real and virtual worlds [11].

The two groups behaved differently with regard to the two conditions. While normal subjects did not exhibit more signs of cybersickness in AVis condition, the level of discomfort of patients was significantly higher in this condition. The relationship between the different anxiety scores and cybersickness underlines the high sensitivity to sensory conflict in anxious patients.

These results are in agreement with the hypothesis of a multisensory integration deficit in this population [32]. It remains to be shown whether the relationship between anxiety and cybersickness is linked specifically to the involvement of auditory modality. Indeed, noise is one of the main contributors to the sensory overload characterizing urban environments, and impaired performances at the visual recognition test in AVis condition could be attributed to this sensory overload [17]. Altogether, the attempt to continuously adjust the relative weighting of auditory, visual and idiothetic information may have caused an attentional load which prevented allocation of attentional resources to the VE.

In the set-up we used, the imperfect mapping between the motor outflow and the multiple sensory feedbacks (movement of the head and its visual and auditory feedbacks) can also be the cause of the increased symptoms of cybersickness in the AVis condition. It exhibits the significance of mastering the delay between sound and images updating so that no supplementary conflict is introduced. A synergistic multimodal VR system should be constructed, allowing modality synchronization through perfect temporal alignment.

The present experiment confirmed the importance of 3-D audio for the construction of a virtual space. Control subjects said that the experience was more compelling when 3-D auditory information was delivered during the virtual navigation, while several patients reported that the two worlds (auditory and visual) could not fulfill a sense of realism when presented together. The visual world we were using was composed of rich textures attempting to model a realistic urban environment. The auditory world was mainly composed of ambisonic sounds recorded in a city, which corresponded to highly textured sounds. In spite of the equivalent richness of both channels, anxious patients tended to perceive them separately.

If this mode of perception can be linked to a sensory overload originating from their high sensitivity to multisensory information, a question remains about the sensation of coherence at the semantic level between the visual scene and the auditory scene [31]. Focusing on this issue, we are currently conducting an experiment in which the visual environment is purely symbolic. Assuming that patients would not have a dual mode of perception in a symbolic VE, it might be possible to unravel primitives that might be sufficient to elicit emotional reactions, presence and rehabilitation.

Computationally it is currently easier to achieve high resolution and realism in an auditory VE than in a visual VE. In an attempt to address this issue, we conducted a trial on a subset of four patients who were asked to navigate blindfolded in an auditory only VE and were surprised to observe that time of immersion tremendously increased (patients were willing to explore the VE as long as possible) while navigation was efficient (all auditory landmarks were found). Furthermore, realism was judged as very high and patients produced an accurate graphic reproduction of layout of auditory landmarks. This condition seems therefore promising for research in therapeutic methods in which VR should not limit its aim to copy reality but should invent new ways to engage the immerged subject.

Applied research with virtual sound have been performed for the last decade in order to allow the visually impaired to develop more accurate and extensive knowledge of spatial 
layout $[9,19]$. Hopefully this kind of VR will provide therapeutic benefit for several psychopathological disorders as well.

\section{Conclusion}

The present study exhibited that presence is increased with 3-D sound in a population of agoraphobic patients, which suggests that this modality is worth being integrated in a VR set-up. However, it demonstrated that agoraphobic patients were more sensitive to the sensory conflict created by the addition of 3-D sound, since cybersickness scores increased tremendously in this condition. Sensory overload might have been the cause of a decrease in spatial performances as assessed after AVis navigation. Further studies should be conducted to integrate efficiently 3-D sound in VR set-up for clinical use, together with studies focusing on 3-D sound, with VR set-up not mediating vision, which might represent a very powerful condition for anxious patients.

\section{Acknowledgements}

We are grateful to Olivier Delerue for providing ListenSpace, the software which enabled the authoring of soundscapes, and Guillaume Vandernoot for his work on HRTFs measurements. We thank Ludivine Sarlat, Feryel Znaïdi, Antoine Pelissolo and Johana Santos for their help with the patients. This study was supported by the French program "Cognition et traitement de l'information" from the CNRS, grant CTI 01-54. Reprint requests to Isabelle Viaud-Delmon, CNRS UMR 7593, Hôpital de la Salpêtrière, Pavillon Clérambault, 47 bd de l'Hôpital, 75013 Paris, France.

\section{References}

[1] Bernstein EM, Putnam FW. Development, reliability, and validity of a dissociation scale. J Nerv Ment Dis 1986;174:727-35.

[2] Blauert J. Spatial hearing. Cambridge: MA: MIT Press; 1983.

[3] Botella C, Banos RM, Perpina C, Villa H, Alcaniz M, Rey A. Virtual reality treatment of claustrophobia: a case report. Behav Res Ther 1998;36:239-46.

[4] Bremner JD, Staib LH, Kaloupek D, Southwick SM, Soufer R, Charney DS. Neural correlates of exposure to traumatic pictures and sound in Vietnam combat veterans with and without posttraumatic stress disorder: a positron emission tomography study. Biol Psychiatry 1999;45:806-16.

[5] Carlin AS, Hoffman HG, Weghorst S. Virtual reality and tactile augmentation in the treatment of spider phobia: a case report. Behav Res Ther 1997;35:153-8.

[6] Darves-Bornoz JM, Degiovanni A, Gaillard P. Validation of a French version of the Dissociative Experiences Scale in a rape-victim population. Can J Psychiatry 1999;44(3):271-5.

[7] Delerue O, Warusfel O. Authoring of virtual sound scenes in the context of the LISTEN project. In: Proceedings of the 22nd AES Conference. 2002. p. 39-47.
[8] Gilkey RH, Weisenberger JM. The sense of presence for the suddenlydeafened adult: Implications for virtual environments. Presence Teleoper Virtual Environ 1995;4(4):357-63.

[9] Golledge RG, Loomis JM, Klatzky RL, Flury A, Yang X. Designing a personal guidance-system to aid navigation without sight: progress on the GIS component. Int J Geogr Inform Syst 1991;5:373-95.

[10] Hendrix C, Barfield W. The sense of presence within auditory virtual environments. Presence Teleoper Virtual Environ 1996;3:290-301.

[11] Ichimura A, Nkajia I, Juzoji H. Investigation and analysis of a reported incident resulting in an actual airline hijacking due to a fanatical and engrossed VR state. Cyberpsychol Behav 2001;4:355-63.

[12] Jacob RG, Furman JM, Durrant JD, Turner SM. Panic, agoraphobia, and vestibular dysfunction. Am J Psychiatry 1996;153:503-12.

[13] Jacob RG, Furman JM, Durrant JD, Turner SM. Surface dependence: a balance control strategy in panic disorder with agoraphobia. Psychosom Med 1997;59:323-30.

[14] Jot JM. Real-time spatial processing of sounds for music, multimedia and interactive human-computer interfaces. Multimedia Syst 1999;7: $55-69$.

[15] Jot JM, Warusfel O. A real-time spatial sound processor for music and virtual reality applications. In: Proceedings of ICMC'95. Canada: Banff; 1995. p. 294-5.

[16] Lecrubier Y, Sheehan D, Weiller E, Amorim P, Bonora LI, Harnett K, et al. The Mini International Neuropsychiatric Interview (MINI). A short diagnostic structured interview: reliability and validity according to the CIDI. Eur Psyhichiatry 1997;12:224-31.

[17] Lipowski ZJ. Sensory and information inputs overload: behavioural effects. Comp Psychiatry 1975;16:199-221.

[18] Loomis JM. Distal attribution and presence. Presence Teleoper Virtual Environ 1992;1(1):113-9.

[19] Loomis JM, Klatzky RL, Golledge RG. Navigating without vision: basic and applied research. Optom Vis Sci 2001;78:282-9.

[20] Marriage J, Barnes NM. Is central hyperacusis a symptom of 5-hydroxytryptamine (5-HT) dysfunction? J Laryngol Otol 1995;109: 915-21.

[21] Moore K, Wiederhold BK, Wiederhold MD, Riva G. Panic and agoraphobia in a virtual world. Cyberpsychol Behav 2002;5(3):197-202.

[22] Reason JT. Relations between motion sickness susceptibility, the spiral after-effect and loudness estimation. Br J Psychol 1968;59:38593.

[23] Regenbrecht HT, Schubert TW, Friedman F. Measuring the sense of presence and its relation to fear of heights in virtual environments. Int J Hum Comput Interact 1998;10:233-49.

[24] Robillard G, Bouchard S, Fournier T, Renaud P. Anxiety and presence during VR immersion: a comparative study of the reactions of phobic and non-phobic participants in therapeutic virtual environments derived from computer games. Cyberpsychol Behav 2003;6(5):46776.

[25] Rothbaum BO, Hodges LF, Kooper R, Opdyke D, Williford JS, North M. Effectiveness of computer-generated (virtual reality) graded exposure in the treatment of acrophobia. Am J Psychiatry 1995;152: $626-8$.

[26] Schubert T, Friedmann F, Regenbrecht H. The experience of presence: factor analytic insights. Presence Teleoper Virtual Environ 2001;10: 266-81.

[27] Sheridan TB. Musings on telepresence and virtual presence. Presence Teleoper Virtual Environ 1992;1(1):120-5.

[28] Spielberger CD, Gorsuch RL, Lushene R, Vagg PR, Jacobs GA. Manual for the State-Trait Anxiety Inventory (STAI), Form Y. Palo Alto: Consulting Psychologists Press; 1983.

[29] Stephens SD. Personality and the slope of loudness function. Q J Exp Psychol 1970;22:9-13. 
[30] Tarr MJ, Warren WH. Virtual reality in behavioral neuroscience and beyond. Nat Neurosci 2002;5:1089-92.

[31] Vastfjall D, Larsson P, Kleiner M. Emotion and auditory virtual environments: affect-based judgments of music reproduced with virtual reverberation times. Cyberpsychol Behav 2002;5:19-32.

[32] Viaud-Delmon I, Ivanenko YP, Berthoz A, Jouvent R. Adaption as sensorial profile in trait anxiety: a study with virtual reality. J Anxiety Disord 2000;14:583-601.
[33] Viaud-Delmon I, Berthoz A, Jouvent R. Multisensory integration for spatial orientation in trait anxiety subjects: absence of visual dependence. Eur Psychiatry 2002;17:194-9.

[34] Wiederhold BK, Jang DP, Kaneda M, Cabral I, Lurie Y, May T, et al. An investigation into physiological responses in virtual environments: an objective measurement of presence. In: Riva G, Galimberti C, editors. Towards cyberpsychology: Mind, cognitions and society in the internet age. Amsterdam: IOS Press; 2003. p. 175-83.

[35] Yardley L, Luxon L, Bird J, Lear S, Britton J. Vestibular and posturographic test results in people with symptoms of panic and agoraphobia. J Audiological Med 1994;3:48-65. 